\title{
ADJSA: An Adaptable Dynamic Job Scheduling Approach Based on Historical Information
}

\author{
(Work-in-Progress) \\ ${\text { Lan } \mathrm{Xu}^{1,2} \text { Qiao-ming Zhu }}^{1,2}$ Zhengxian Gong ${ }^{1,2}$ Pei-feng $\mathrm{Li}^{1,2}$ \\ ( ${ }^{1}$ School of Computer Science \& Technology, Soochow University, Suzhou, China, 215006) \\ ( ${ }^{2}$ Key Lab of Computer Information Processing Technology of Jiangsu Province, Suzhou, China, 215006)
}

Abstract: Currently, there are many researches focusing on grid scheduling and more and more scheduling algorithms were proposed. However, those algorithms are not satisfied with the requirement of the grid for ignoring its characteristics of dynamics, autonomy, distributing, etc. Therefore, this paper proposes an adaptable dynamic job scheduling approach based on historical information (ADJSA). This approach adjusts the predicting model automatically by using the recent jobs execution historical information and then selects the appropriate resource to execute the job considering dynamic and real-time factors of the Grid.

Key words: grid computing; job scheduling; historical information; adaptation

\section{Introduction}

Due to the nature of resources in grid which is dynamic, distributed and autonomic, the job scheduling in grid is more challenging than traditional scheduling. At its essence, scheduling is an optimization problem because only a finite number of resources are available in a grid, and there may be more work requests than its capacity. The job scheduling is a NP-problem and a lot of research has been made on it. Nowadays, it is still one of hot issues in grid research.

Maheswaran et al. [1] categorized job scheduling into "static" job scheduling and "dynamic" job

Permission to make digital or hard copies of all or part of this work for personal or classroom use is granted without fee provided that copies are not made or distributed for profit or commercial advantage and that copies bear this notice and the full citation on the first page. To copy otherwise, or republish, to post on servers or to redistribute to lists, requires prior specific permission and/or a fee.

INFOSCALE 2007, June 6-8, Suzhou, China Copyright (C) 2007 ICST 978-1-59593-757-5

DOI 10.4108/infoscale.2007.977 scheduling. Static scheduling ${ }^{[2]}$ is the scheduling such that all decisions are done before the execution of a schedule and at the expense of the real-time property of single job. In contrast, dynamic scheduling ${ }^{[3]}$ is the scheduling such that some or all decisions are done during the execution of a schedule and consider the real-time property of single job. However, dynamic scheduling ignores the influence of performance evaluation caused by statistical information of job and resources and the job execution. So, dynamic scheduling is more suitable for the grid environment than static scheduling. For these, ADJSA adopts the dynamic scheduling policy which can be fit for the dynamics and autonomy of grid well.

\section{Approach}

ADJSA consider the job scheduling in grid from the following three points:

(1) The reliable information of grid resource. The reliability of resource (Re) indicates the successful probability of job executed on this resource. For users, the $\mathrm{Re}$ represents the possibility of successful execution on the resource. ADJSA represents Re using Eq. (1) :

$$
\begin{aligned}
& \operatorname{Re}(X)=\frac{1}{n} \sum_{i=1}^{n} \text { succ }_{i} \\
& \text { succ }_{i}= \begin{cases}1 & \text { job executed successfully } \\
0 & \text { job executed unsuccessfully }\end{cases}
\end{aligned}
$$

(2) The historical information of job. It is necessary to show that the duration of jobs can be predicted more accurately when historical information is used than when it is not. But the type of grid job is so multiple that is a great challenge to prediction work. The research in this paper emphasizes on how to utilize the historical information to predict the execution time of current job. ADJSA forecasts the execution time using Eq. (2): 
$T_{e s t}^{n}=\gamma T_{e s t}^{n-1}+(1-\gamma)\left(T_{e x c}^{n-1}+\beta_{n-1}\right)$

$\beta_{n}=\sqrt{\frac{1}{n-1}\left(\sum_{i=1}^{n}\left(T_{e \times c}^{i}\right)^{2}-\frac{\left(\sum_{i=1}^{n} T_{e \times c}^{i}\right)^{2}}{n}\right)}$

where $T_{e s t}^{n}$ denotes the estimated time of the n-th job on a resource, $\beta_{n}$ denotes the standard deviation which reflect the historical condition of job execution on this resource. $T_{e s t}^{n-1}$ shows the influence of historical predication on current time predication, while $\left(T_{e x c}^{n-1}+\beta_{n-1}\right)$ presents that of historical actual execution. And $\gamma$ is the coefficient to control the weights of above two parts. $\gamma$ is adjusted dynamically in terms of $\varphi$ defined by virtue of $\varphi=\frac{T_{e x c}-T_{e s t}}{T_{e x c}}$.

ADJSA sets the value of $\gamma_{\text {based }}$ on the value of $\varphi$ and can obtain the following criteria:

$\gamma= \begin{cases}\frac{T_{e x c}}{T_{e s t}}, & T_{e s t}>2 T_{e x c} \\ \frac{T_{e s t}}{T_{e x c}}, & T_{e s t} \leq T_{e x c} \\ 1-\frac{T_{e x c}}{T_{e s t}}, & T_{e x c}<T_{e s t} \leq 2 T_{e x c}\end{cases}$

It can be shown that the value of $T_{\text {est }}$ and $T_{e x c}$ of last job execution result in the influence of feedback on next prediction and $\gamma$ is changed dynamically to adjust the performance of predicting model.

(3) The real-time information of resources

(Uncertainties). ADJSA considers the uncertainties on the organic combination of historical and real-time information (Eq.(4)).

$M_{p}=\rho\left(1-C p u_{\text {load }}\right)+(1-\rho)\left(1-N_{\text {load }(a, b)}\right) \quad 0<\rho<1$

Let $C p u_{\text {load }}$ denotes the current Cpu load of resource, Net $_{\text {load (a,b) }}$ denotes the network load between node a and node b. $\rho$ is to control the weights of Cpu load and network load. The Eq.(4) shows the overall load of current resource and can balance the workload of system effectively validated by the subsequent experiments.

ADJSA defines the performance value of resource as $P_{v a l}$ by virtue of

$$
P_{v a l}=\operatorname{Re}(x) \times \frac{M_{p}}{T_{\text {est }}}
$$

$\mathrm{P}_{\mathrm{val}}$ can reflect the overall status of resource well on a synthetic consideration of historical and real-time information of resource. In the actual implementation, the
PerService which is a web service to notify the status report of resource to the scheduler node periodically is deployed in every resource node in grid (the periodic time is set to $60 \mathrm{~s}$ in our environment). If a report exceeds the effective deadline existed in every status report, the node associated with the overtime report is set invalidation (the effective deadline is defined as $\left.\mathrm{T}_{\min }=3^{*} 60 \mathrm{~s}\right)$. As a result, the invalid node can't be selected during the job scheduling process.

\section{Conclusion}

This paper focuses on job scheduling algorithm under the dynamic environment with various uncertainties such as CPU load, network traffic etc. Meanwhile, it points out disadvantages of current scheduling algorithms and proposes an adaptable dynamic job scheduling approach -ADJSA. Due to think of the CPU load and network load of a resource, ADJSA can improve throughput and balance the workload of total grid system. Moreover, ADJSA makes use of the Notifications mechanism that the performance report of each resource will notify the scheduler periodically. So scheduler needn't to query the information service (eg. MDS) on each node and submit job to the node as possible. In our experiments, ADJSA is shown to have favorable job scheduling effects.

\section{Acknowledgments}

This research was supported by the National Natural Science Foundation of China under Grant No. 60673041 and Nation 863 project of China under Grant No. 2006AA01Z147 and the High Technology Plan of Jiangsu Province of China under Grant No. 2005030.

\section{References}

[1] Maheswaran M, Ali S, Siegel HJ, Hensgen D, Freund RF. A comparison of dynamic strategies for mapping a class of independent jobs onto heterogeneous computing systems. Technical Report, School of ECE, Purdue University, 1999

[2] Yang Y, Cai ZX, Fu Y, Liu MQ. An adaptive grid job scheduling method based on genetic algorithm[J]. Computer Engineering And Applications, 2005.1

[3] William K. Cheung, Jiming Liu, Kevin H. Tsang, Raymond K. Wong, Dynamic resource selection for service composition in the grid. IEEE/WIC/ACM International Conference on Web Intelligence(WI’04), 2004 